\title{
Relative Binding Free Energy Predictions for Inhibitors of Tetrameric Influenza Virus Neuraminidase
}

\author{
Billy J. Williams-Noonan, Elizabeth Yuriev ${ }^{*}$ and David K. Chalmers*
}

\begin{abstract}
Medicinal Chemistry, Monash Institute of Pharmaceutical Sciences, Monash University, 381 Royal Pde Parkville, Victoria 3052, Australia.
\end{abstract}

\begin{abstract}
Accurate methods to predict the free energies of protein-ligand interactions have great potential to assist rational drug design. In this work, we used molecular dynamics simulations with alchemical perturbation to predict the binding of carbohydrate-based ligands to influenza virus neuraminidase (N2). This specific drug target is a challenging test system for alchemical free energy methods because it has flexible binding site motifs. We use a molecular dynamics protocol that works for longer time scales than are often reported in previous molecular dynamics studies of N2. We demonstrated that N2-ligand complex stability and that accurate N2 150-loop dynamics, on a $350 \mathrm{~ns}$ time scale, are both force fielddependent (AMBER99SB-ILDN, GAFF and TIP4P water are an optimal combination). Further, we showed that crystallographic waters must be retained to maintain stability of N2-ligand complexes over $350 \mathrm{~ns}$. Using our modelling protocol, we were able to determine relative binding free energy values between neuraminidase ligands which correlated strongly with experimental differences in pIC50 values $(R=-0.96, \rho=0.86, N=13, \mathrm{sig}<0.0001)$. It is anticipated that the molecular dynamics protocol and the relative binding free energy methods reported here, will both be useful in expediting the discovery of novel therapeutics for $\mathrm{N} 2$ and other homologous glycohydrolases.
\end{abstract}

\section{INTRODUCTION}

Rational drug design is underpinned by an understanding of the interaction s between a potential drug and its biological target. In some projects, the nature of the interactions may be only poorly understood while in others, the interactions can be well characterised through crystallography or other methods that provide a detailed, atomic scale picture of the ligand in the binding site. However, even when good structural information is available, it is frequently difficult to use this information to design more potent compounds. With the development of computational power and improvements in algorithms, in silico free energy methods have expanded potential to guide the development of biologically active 
compounds, by affording estimates of protein-ligand interaction strength. Here, we conduct a retrospective study, to investigate the ability of alchemical free energy methods to reproduce experimental measures of binding strength for the glycohydrolase enzyme neuraminidase, which is a key virulence factor in the influenza virus. We provide here an optimal simulation protocol for neuraminidase-ligand complexes and complement existing evidence that molecular modelling methods have scope in assisting the design of novel anti-influenza drugs, as reviewed by Patel et al. ${ }^{1}$

It is common to rank compounds by binding affinity, in terms of the free energy difference between the 'bound' and 'free' states $\left(\Delta G_{b i n d}\right)$. Binding affinity is often expressed terms of the dissociation constant, $K_{D}$ (Equation 1) where $[P],[L]$ and $[P L]$ are the concentrations of the protein, ligand and complex. The free energy of binding is related to the $K_{D}$ as shown in Equation 2, $R$ is the gas constant and $T$ is the absolute temperature. ${ }^{2}$

$$
\begin{aligned}
& K_{D}=\frac{[P][L]}{[P L]} \\
& \Delta G_{b i n d}=R T \ln K_{D}
\end{aligned}
$$

Since many physical events (e.g. protein-ligand binding) occur outside the timescales that are easily accessible by MD, Zwanzig proposed that a system could be perturbed between two different states, but with respect to the variable $\lambda$, instead of time. ${ }^{3}$ Methods that do this are referred to as 'alchemical'. The original alchemical perturbation method is 'Free Energy Perturbation' (FEP), ${ }^{3}$ where the phase space between two states is sampled with respect to the scaling variable $(\lambda)$. The system is in state A when $\lambda$ $=0$, the, and in state B when $\lambda=1$. As shown in Figure 1, this methodology can be used to calculate the difference in free energies of binding $(\Delta \Delta G)$ of two ligands, $\mathrm{A}$ and $\mathrm{B}$. This approach is commonly known as a relative free energy calculation. In this instance, state $\mathrm{A}$ includes ligand $\mathrm{A}$, and state $\mathrm{B}$ uses ligand B. Two series of simulations are performed where ligand A is non-phy sically transformed into ligand B by addition/deletion of atoms, transformation of atoms from one element to another or modification of bonds (e.g. transformation of single to double bonds). One set of transformation calculations is performed with the ligand in solution and the other with the ligand in the binding site. By subtraction of the free energy changes in the binding site and in solution, one can calculate the difference in binding free energies of ligands A and B (Figure 1). In a recent study, ${ }^{4}$ we compared a number of methods for predicting the binding affinity of ligands. In that work, we found the relative alchemical perturbation method to be most accurate method and of sufficient accuracy to be useful in drug development. Relative alchemical perturbation methods are increasingly being applied in industrial and academic research settings. ${ }^{5-7}$ 
Relative Free Energy by Perturbation

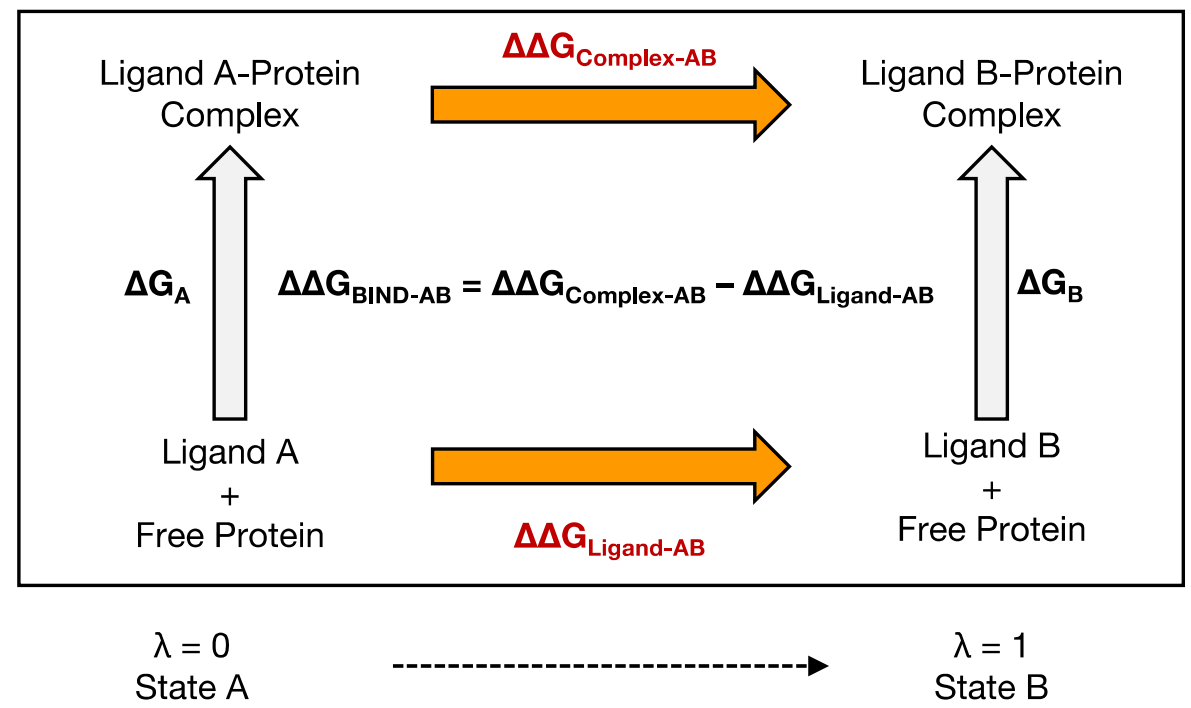

Figure 1.Prediction of binding free energies using the relative method. Arrows depicting the perturbation reaction being simulated over a series of $\lambda$-states are in grey. Arrows forlong time-scale reactions are in yellow.

Influenza is a debilitating disease with a high mortality rate and there have been multiple influenza virus pandemics in the past century. ${ }^{8}{ }^{9}$ Influenza has been successfully targeted by a number of antiinfluenza therapeutics including Relenza (zanamivir) ${ }^{10,11}$ and Tamiflu (oseltamivir) ${ }^{12-15}$. Relenza and Tamiflu are ester prodrugs that are cleaved to the active forms, Relenza carboxylate and Tamiflu carboxylate (Figure 2), following absorption. The free acids act by inhibiting the influenza virus enzyme neuraminidase, a glycohydrolase that cleaves 5- $\mathrm{N}$-acetyl neuraminic acid (Neu5Ac) residues from cell surface glycosides. Other inhibitors of neuraminidase include Neu5Ac2en and glucuronic acid (GlcA) derivatives combine the isopentyl ether group of Tamiflu with the unsaturated pyranose scaffold of Relenza (Figure 2).

Neuraminidase plays a crucial role in viral replication in allowing newly synthesised viral progeny to escape the host cell. Inhibition of viral neuraminidase is a validated mechanism for targeting influenza virus. Blocking the active site of influenza virus neuraminidase means that the viral progeny remain attached to the host cell, and the host immune system is then able to remove the trapped virions. There are 9 subtypes of neuraminidase (N1 to N9). ${ }^{16,17}$ All subtypes of viral neuraminidase exist as tetramers, ${ }^{18}$ as shown in Figure 3A. An illustration of the influenza virus neuraminidase active site with Tamiflu bound is shown in Figure 3B. The overlay of crystal structures of all neuraminidase subtypes reveals very similar binding site architecture among each variant of neuraminidase. The active site contains two flexible protein motifs. Conformational change in the 150-loop occurs when inhibitors of neuraminidase bind to the active site. Specifically, the volume underneath the 150-loop (the 150-cavity, Figure 3B) expands to allow for the binding of bulkier substituents. Besides the global SARS-CoV-2 pandemic that continues into 2021, otherrecent pandemics have been caused by the influenza virus containing the following hemagglutninin and neuraminidase subtypes: H1N1 (in 1918), H2N2 (1957), H3N2 (1968) 
and H5N1 (in 2006). ${ }^{8,9}$ Therefore, the N1 and N2 subtypes of neuraminidase are the most relevant with respect to human disease.

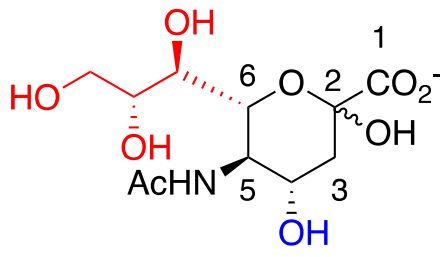

5- $\mathrm{N}$-acetyl neuraminic acid (Neu5Ac)

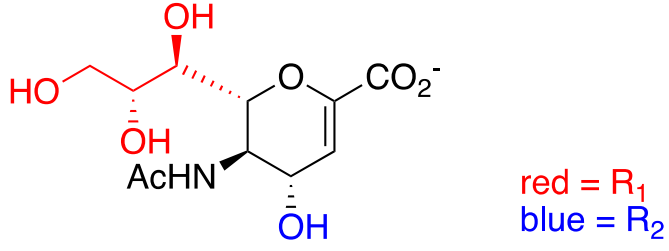

Neu5Ac2en<smiles>NC(=[NH2+])N[C@H]1C=C(C(=O)[O-])O[C@H]([C@H](O)[C@H](O)CO)[C@H]1N</smiles>

Relenza carboxylate<smiles>[R2]C1C=C(C(=O)[O-])O[C@@H](OC(CC)CC)[C@@H]1NC</smiles>

glucuronic acid (GlcA) analogs<smiles>CCCC(CC)O[C@H]1C=C(C(=O)[O-])C[C@H]([NH3+])[C@H]1NC</smiles>

Tamiflu carboxylate (1)

Figure 2. Structures of 5-N-acetyl neuraminic acid and (Neu5Ac) and neuraminidase inhibitors.

A

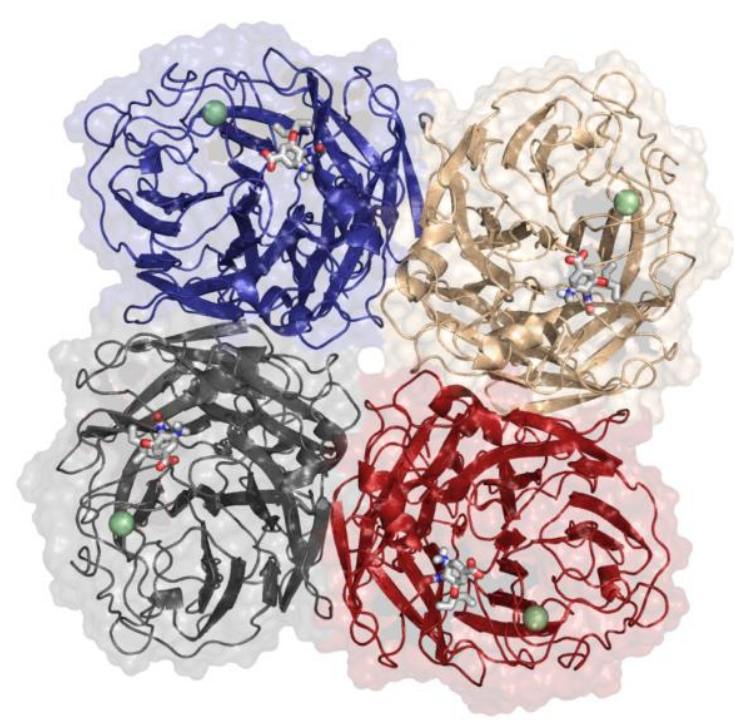

B

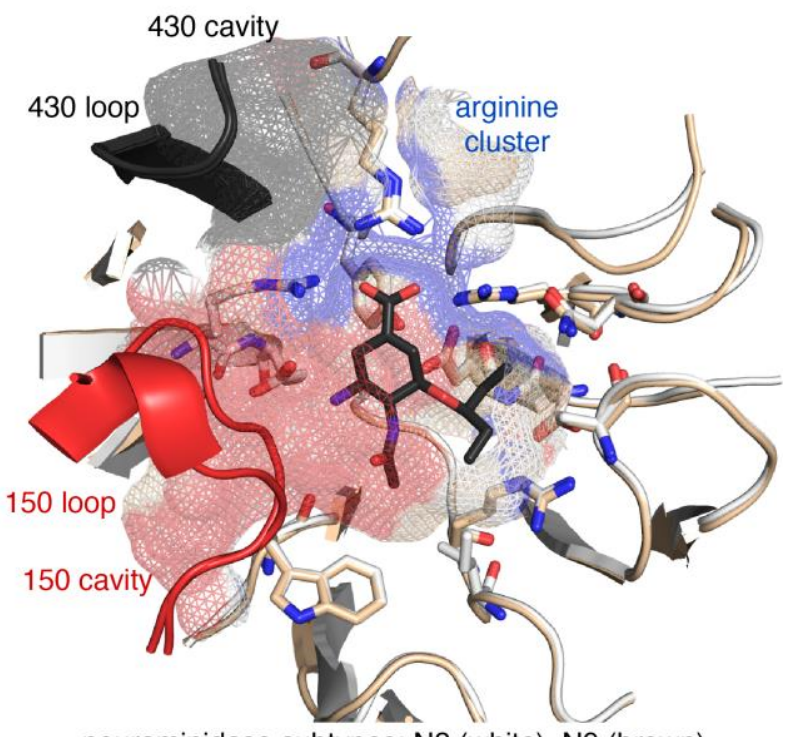

neuraminidase subtypes: N2 (white), N9 (brown)

Figure 3. (A) The N2 neuraminidase tetramer from PDB-ID 4D8S. ${ }^{19}$ Protein chains are shown as ribbons with transparent surfaces. Calcium ions in the active site of each monomer are shown as green spheres. Bound inhibitors are shown as sticks with grey carbon, white hydrogen, red oxygen and blue nitrogen. (B) Overlay of the binding sites of two neuraminidase subtypes: N2 (PDB-ID: 4K1K, white) ${ }^{20}$ and N9 (PDB-ID: 6HEB, light brown). The ligand Tamiflu carboxylate (1) is shown in black. A ribbon representation is given for residues within $12 \AA$ of the 150- and 430-loops framing the active site are shown as ribbons representations coloured red and black. The cavities underneath these loops are referred to as the 150 - and 430 -cavities 
The inhibitors shown in Figure 2 make specific interactions with the active site of influenza virus neuraminidase. Nine conserved residues in the neuraminidase active site make contact with Neu5 Ac2enbased inhibitors. ${ }^{10,11,19,20}$ A cluster of three arginine residues in the active site (Arg-118, Arg-272 and Arg-371) interact with the carboxylate group of Neu5 $\mathrm{Ac}^{21}$ and related ligands. When $\mathrm{R}_{1}$ (red, Figure 2) is a glycerol side chain (e.g. in Relenza carboxylate), the hydroxyl groups of this moiety make hydrogen bonding interactions with Glu-276 and active site water molecules. Tamiflu-like ligands contain an isopentyl ether group as $\mathrm{R}_{1}$, which binds to neuraminidase by an induced-fit effect, where Glu-276 in the receptor flips to interact with Arg-224, and active site waters are displaced so that the isopentyl ether can make hydrophobic interactions with the receptor. ${ }^{19,20}$ The $\mathrm{R}_{2}$ group (blue, Figure 2) projects into a pocket covered by the active site 150-loop (Figure 3B). This protein loop is flexible, and shifts to accommodate the bulkier 4-guanidino group of Relenza-like ligands. The 4-hydroxy group of Neu5Ac2en interacts favourably with Glu-119. Likewise, the 4-amino group of Tamiflu carboxylate forms a salt bridge between Glu-119, ${ }^{19},{ }^{20}$ while the guanidinium of Relenza carboxylate interacts with Glu-119 and Glu-227 (Figure 3B). ${ }^{10,11}$

In 2012, Bhatt et al. demonstrated that unsaturated glucuronic acid (GlcA) analogues inhibit neuraminidase with low to mid $\mu \mathrm{M}$ IC50 values (Table 1 ).${ }^{19}$ It was shown by NMR that the modest affinity of these inhibitors is explained by differences between conformations of the GlcA ligand in solution and the bound GlcA ligand. ${ }^{19}$ The energetic penalty associated with adopting the bound conformation explains the weaker binding affinity for ligands 2-6 compared with Tamiflu carboxylate or Relenza carboxylate, which are sub-nanomolar binders to neuraminidase. 
Table 1. IC50 values for neura minida se inhibition by inhibitors with Tamiflu and glucuronic acid (GlcA) scaffolds.

\begin{tabular}{|c|c|c|c|c|c|}
\hline Compound & $\mathbf{X}$ & $\mathbf{Y}$ & $\begin{array}{l}\text { Neuraminidase } \\
\text { IC50 }(\mathrm{nM})\end{array}$ & & pIC50 $^{d}$ \\
\hline 1 & $\mathrm{H}$ & - & $0.37(0.09)^{\mathrm{a}}$ & 9.43 & $(9.26-9.72)$ \\
\hline 2 & - & $\mathrm{OH}$ & $320(40)^{\mathrm{b}}$ & 6.49 & $(6.40-6.62)$ \\
\hline 3 & - & $\mathrm{CH}_{2} \mathrm{CH}_{3}$ & $28000(3600)^{b}$ & 4.55 & $(4.45-4.68)$ \\
\hline 4 & - & $\mathrm{CH}_{3}$ & $1450(190)^{\mathrm{b}}$ & 5.84 & $(5.74-5.97)$ \\
\hline 5 & - & $\mathrm{CH}_{2} \mathrm{CH}=\mathrm{CH}_{2}$ & $212000(28000)^{\mathrm{b}}$ & 3.67 & $(3.57-3.81)$ \\
\hline 6 & - & $\mathrm{CH}_{2} \mathrm{C}(=\mathrm{O}) \mathrm{CH}_{3}$ & $5390(700)^{b}$ & 5.27 & $(5.17-5.40)$ \\
\hline 7 & $-\mathrm{C}(=\mathrm{O}) \mathrm{H}$ & - & $1290(110)^{\mathrm{c}}$ & 5.89 & $(5.82-5.97)$ \\
\hline 8 & $-\mathrm{C}(=\mathrm{O}) \mathrm{CH}_{3}$ & - & $240(10)^{\mathrm{c}}$ & 6.62 & $(6.59-6.66)$ \\
\hline 9 & $\mathrm{C}(=\mathrm{O}) \mathrm{CH}=\mathrm{CH}_{2}$ & - & $2980(210)^{\mathrm{c}}$ & 5.53 & $(5.47-5.59)$ \\
\hline
\end{tabular}

aFrom Gubareva et $^{2} \mathrm{l}^{22}$ mea sured against N2 neura minidase. Number in brackets is a standard deviation (SD) from triplicate experiments. ${ }^{b}$ From Bhatt et $a l^{19}$ measured against N2 neuraminidase. The SD values in brackets is a pproximated from information given by Bhatt $e t a l .{ }^{c}$ From Alexander et $a l^{23}$ measured against N1 neuraminidase. Standard deviation in brackets is from the paper by Alexander et al. ${ }^{\mathrm{d}}$ The pIC50 values are determined from the molar IC50. Numbers in brackets are a range of pIC50 values, spanning \pm 2 SD in IC50.

One of the first applications of molecular dynamics to predict binding affinities to neuraminidase was reported in a study by Taylor and von Itzstein in $1994 .{ }^{24} \mathrm{Neu} 5 \mathrm{Ac}$, Neu5Ac2en, 4 -amino Neu5Ac2en and Relenza carboxylate were docked into the binding site of N9 neuraminidase and a series of energy minimisations and short MD simulations were then run to produce low energy structures for the complexes. By analysing these structures and measuring the distances between interacting residues in the binding site and the ligand, the binding enthalpies were predicted. These binding enthalpies were reported to correlate with experimental measurements of binding strength.

More recently, a variety of free energy methods have been applied to predict binding of neuraminidase ligands. Using the thermodynamic integration method,Lawrenz et al. ${ }^{25}$ predicted the binding free energy of the inhibitor peramivir binding to the $\mathrm{N} 1$ neuraminidase tetramer to within $1.1 \mathrm{~kJ} \mathrm{~mol}^{-1}$ of the experimental value. They sampled bound conformations using replicatesimulations with the GROMOS96 force field, totalling to $800 \mathrm{~ns}$ of simulation time. In a separate study, ${ }^{26}$ they compared the ability of the GROMOS-96 and AMBER-99SB force fields to predict binding free energies for two neuraminidase ligands, in the presence and absence of binding site calcium ions. They found that the GROMOS-96 and AMBER-99SB force fields performed comparably well in predicting binding strength, and the presence of the binding site calcium ion did not significantly affect inhibitor binding strength. 
Simulations where biasing forces are applied to pull a ligand from the binding site are referred to as steered simulations. The maximum force required to pull a ligand from a binding site is commonly referred to as $F_{\max }$, or the rupture force. Mai et al. used the AMBER-99SB force field and showed that $F_{\max }$ correlated with experimental binding free energies for monomeric N1 neuraminidase, with $R=$ $0.70 .{ }^{27}$ In a separate study, ${ }^{28}$ the same authorsfound a correlation between $F_{\max }$ and $\Delta G_{\text {bind }}$ for 24 proteinligand complexes involving variants of neuraminidase, with $R=0.97$. Two novel anti-viral agents were included in the latter study, one of which (CS-8958) has shown efficacy both in vitro and during clinical trials. $^{27,29,30}$ In these two studies, Mai et al. ${ }^{27,28}$ compared steered MD to the MM-PBSA method and found that steered MD was more accurate for influenza virus neuraminidase inhibitors.

Nguyen et al. ${ }^{31}$ also performed MM-PBSA endpoint calculations on neuraminidase ligands trialling a series of different protein force fields and water models. All simulations were performed with tetrameric neuraminidase. The authors found that the predicted binding free energy data correlated strongly with the density of water molecules in the active site $(R=0.90)$, using the AMBER-99SB force field and TIP4P water molecules. The use of the OPLS force field also gave a good correlation between active site water density and the ligand binding free energy $(R=0.67)$ while the CHARMM and GROMOS (where only two ligands in the set were predicted to bind) did not model protein-ligand complex stability well. This suggests that force field choice and the presence of water molecules in the active during a simulation has an effect on the predicted binding free energy.

Amaro et al. $^{32}$ investigated the dynamics of the active site 150- and 430-loops with respect to the N1 and N2 variants of neuraminidase. They ran $100 \mathrm{~ns}$ molecular dynamics simulations using an AMBER force field and a TIP4P water model with tetrameric neuraminidases. The 150-loop was maintained in position by a salt bridge between Asp-147 and His-150. Importantly, this ionic lock was observed to break intermittently over the short $100 \mathrm{~ns}$ timescale, resulting in changes to the 150 -cavity volume. Simulations with neuraminidase inhibitors bound in the active site were not reported.

Recently, there is a trend toward using simple endpoint methods, such as MM-PBSA, to determine the absolute binding free energy values of ligands binding to wild-type and mutant neuraminidase variants. ${ }^{33-37}$ These simulations have potential in assisting the design of novel anti-influenza drugs specific to wild-type and mutant neuraminidase, with scope in combating anti-viral resistance in influenza virus. Since 2018, authors who have reported the use of endpoint methods to study neuraminidase-ligand binding ${ }^{33-37}$ used different MD protocols and system constructs in each individual study. Notably, Ren et al. ${ }^{34}$ report the use of systems comprising monomeric neuraminidase-ligand complexes for $20 \mathrm{~ns}$ MD simulations, where the trajectories were subject to analysis by MM-PBSA. A total of 16 different forcefield combinations for the protein and water models were compared, in terms of the accuracy of the resulting MM-PBSA calculation. Only 3-point water models were trialled. These authors found that variants of the AMBER forcefield afforded the most accurate measures of proteinligand binding free energy with respect to experiment. 
Together, these studies show that modelling ligands binding to neuraminidase is challenging. In particular, the protein dynamics of the 150-loop must be accurately reproduced by the chosen force in both the free protein and when the protein is in complex with inhibitor. Additionally, the binding site arginine residues make important interactions with the carboxylate of most neuraminidase ligands, and the three arginine side chains are shown to be within $3 \AA$ of each other. Arginine residues are protonated at physiological $\mathrm{pH}$, so the chosen force field must be parameterised such that these positively charged side chains do not repel each other on long timescales.

The aim of this work was to establish a modelling approach that could usefully predict the binding affinities of inhibitors of neuraminidase and potentially other glycohydrolases. In a recent study, ${ }^{5}$ we investigated the ability of a number of free energy methods to predict the binding of ligands to HIV integrase. Of the methods investigated, we found that the relative Bennet's acceptance ration (BAR (relative)) gave the most accurate predictions. We therefore chose to apply this free energy prediction method to neuraminidase. The previous work on neuraminidase modelling, discussed above, has shown that to be successful, we would need to use an appropriate force field, water model, and protein model. It is not clear from previous work what molecular dynamics protocol is best for modelling neuraminidase. In previous studies, modelling has often been performed with tetrameric neuraminidase, ${ }^{31,32}$ but no comparison between monomeric and tetrameric simulations has been reported. Since conformational changes in the active site are important for ligand binding, force field choice will affect binding free energy prediction accuracy if some force fields are better at modelling the binding site flexibility than others. Accordingly, we have used an initial set of unbiased long scale MD simulations to establish a suitable computational model for integrase. Using this model, we have performed BAR (relative) calculations on a series of Tamiflu and GlcA analogues to study the binding of neuraminidase ligands, which we compare to experimental pIC50 values derived from the literature.

\section{METHODS}

Neuraminidase tetramer model: A crystal structure of GlcA (2) (see Table 1) bound to N2 neuraminidase (PDB-ID: 4D8S), with $2.40 \AA$ resolution, ${ }^{19}$ and also a $1.60 \AA$ A resolution structure of N2 neuraminidase with bound Tamiflu carboxylate 1 (PDB-ID: 4K1 K) ${ }^{20}$ were used for modelling. Structure PDB-ID: 4K1K was used to model the protein and binding site water molecules: the glycosylating carbohydrate residues were deleted, missing protein loops modelled with PrimeX, ${ }^{38}$ and a tetrameric model constructed using the symmetry mates. Binding site waters within $10 \AA$ of bound Tamiflu carboxylate were retained. A model of compound 2 bound to neuraminidase was generated by using the Protein Structure Alignment Tool in Maestro 2018.1 to align the proteins from PDB-IDs 4D8S and $4 \mathrm{~K} 1 \mathrm{~K}$ and transferring the GlcA 2 coordinates. The binding site calcium ion was retained. Bound models for ligands 3-6 were manually constructed based on the ligand coordinates of the GlcA 2 model. 
Structures for 7-9 were built from the coordinates of $\mathbf{1}$ bound to N2 from PDB-ID 4K1K. The Protein Preparation Wizard tool in Maestro was used to protonate structures at $\mathrm{pH} 7.0$ and remove clashes. To remove steric strain, these manually built ligands were minimized in Maestro in the presence of the binding site waters and protein to generate predicted bound poses.

MD Simulations: MD simulations were performed using GROMACS 2016.3. ${ }^{39,} 40$ The AMBER99SB-ILDN force field ${ }^{41}$ was used for the protein and ions. The Generalised Amber Force field $(\mathrm{GAFF})^{42}$ was used to parameterize all ligands. Periodic boundary conditions ${ }^{43}$ were set up so that the protein-ligand complex was centred in an $6.0 \times 10.0 \times 10.0 \mathrm{~nm}$ periodic cell. Simulations used a $2 \mathrm{fs}$ time step, and neighbour searching was performed every $80 \mathrm{ps}$. The default Verlet integrator was used. ${ }^{44}$ The LINCS algorithm ${ }^{45}$ was used to maintain rigid bond-lengths. In each case, LINCS-iter was set to 4 and LINCS-order was set to 6. Coulombic and Lennard-Jones potential cut-offs of $1.4 \mathrm{~nm}$ were used for short-range interactions and the Particle Mesh Ewald (PME) ${ }^{46}$ algorithm was used to account for electrostatic interactions beyond this range. The protein-ligand system was solvated to a density of approximately $1.00 \mathrm{~g} \mathrm{~mL}^{-1}$ with explicit TIP4P-Ew water. ${ }^{47}$ The overall charge of the system was made neutral through the addition of sodiumor chlorideions. Additional sodium and chloride ions were added to make a total salt concentration of $\sim 150 \mathrm{mM}$.

Each system was equilibrated using the following procedure. The solvated protein-ligand complex was first subjected to a restrained, 10,000 step, steepest descent energy minimization, with harmonic position restraints $\left(\mathrm{k}=25,000 \mathrm{~kJ} \mathrm{~mol}^{-1} \mathrm{~nm}^{-2}\right)$ on all protein and ligand atoms. This restrained minimisation was followed by an unrestrained 10,000 step steepest descent energy minimization, to relieve any residual steric strain on the system. It was found that the neuraminidase 150-loop and binding site arginine residues were prone to move, and care had to be taken to prevent this during the equilibration phase. The system was therefore equilibrated using series of simulations as shownin Table 2. Throughout the equilibration phases, complexes were monitored for stability using Visu al Molecular Dynamics (VMD). By prior alignment of the trajectory protein coordinates, movement of the ligand by more than $4 \AA$ RMSD from the initial pose was typically associated with unbinding events. Complexes that remained stable for the duration of equilibration phase 8 were then subjected to unbiased MD and alchemical perturbation, using the same ensemble. 
Table 2. Equilibration protocol for neura minida se complexes.

\begin{tabular}{|c|c|c|c|c|c|c|}
\hline Phase & $\begin{array}{c}\text { Time } \\
\text { (ps) }\end{array}$ & Thermostat & $\begin{array}{c}\text { Temp. } \\
\text { (K) }\end{array}$ & Barostat & $\begin{array}{c}\text { Restraint force } \\
\text { constant on protein } \\
\text { heavy atoms } \\
\left(\mathrm{kJ} \mathrm{mol}^{-1} \mathbf{~ m m}^{-2}\right)\end{array}$ & 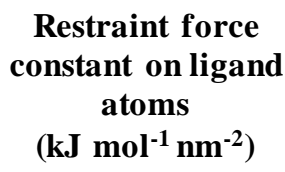 \\
\hline 1 & 300 & Berendsen & 50 & - & 25,000 & 25,000 \\
\hline 2 & 50 & Berendsen & 100 & - & 2500 & 2500 \\
\hline 3 & 50 & Berendsen & 150 & - & 2500 & 2500 \\
\hline 4 & 50 & Berendsen & 250 & - & 2500 & 2500 \\
\hline 5 & 50 & Berendsen & 300 & - & 250 & 250 \\
\hline 6 & 1000 & V-rescale & 300 & Berendsen & 25 & 25 \\
\hline 7 & 300 & V-rescale & 300 & $\begin{array}{c}\text { Parrinello- } \\
\text { Rahman }\end{array}$ & $1^{\mathrm{a}}$ & 1 \\
\hline 8 & 10,000 & V-rescale & 300 & $\begin{array}{c}\text { Parrinello- } \\
\text { Rahman }\end{array}$ & - & - \\
\hline
\end{tabular}

a Restraints on $\mathrm{C} \alpha$ atoms only.

Models for the simulations of the free ligand were constructed in cubic periodic cells with dimensions, $5.0 \times 5.0 \times 5.0 \mathrm{~nm}$. The ligand was solvated to a density of $1.00 \mathrm{~g} \mathrm{~mL}^{-1}$, and water molecules were replaced with $\mathrm{Na}^{+}$or $\mathrm{Cl}^{-}$ions to bring the salt concentration to $150 \mathrm{mM}$ and to neutralize the electric charge of the system. Equilibration of the free ligand in solution began with an unrestrained 10,000 step steepest descent energy minimization. Phases 5, 6 and 8 (Table 2) were used to bring the solvated ligand to a temperature of $300 \mathrm{~K}$ and a pressure of 1.0 bar, except no harmonic position restraints were used, and the protein was not present. Free ligand runs used phase 8 conditions.

BAR (relative): Dual topologies for GROMACS with the AMBER99SB-ILDN force field were constructed with FESetup. ${ }^{48}$ Monomer A of the tetramer had a bound ligand, while the three remaining monomeric units had ligand coordinates removed. A total of 14 relative perturbations were performed between Tamiflu carboxylate (1) and ligands 2-9, whereby one ligand was transformed into another. Each perturbation used $20 \lambda$-states; 10 for the Coulombic interaction perturbation, and then separately, 10 for the vdW radii transformations. Perturbations for the Coulombic and then vdW parameters were performed linearly. Similarly, the bond, dihedral and mass terms were perturbed linearly across the 20 $\lambda$-states. Prior to each simulation, a steepest descent unrestrained minimization was performed at each $\lambda$-state. Each $\lambda$-state simulation was run for $4 \mathrm{~ns}$ for the complex simulations, and $10 \mathrm{~ns}$ for the simulations of the free ligand. The first half of each simulation was used as an additional equilibration phase and analysis was performed on the second half of the simulation. The $\Delta \Delta G$ values were calculated as shown in Figure 1. Hysteresis correction was performed on the closed cycles in the manner described by Wang et al. ${ }^{20}$ with an in-house Python script. 


\section{RESULTS AND DISCUSSION}

In this study, we aimed to predict the binding strengths of complexes of $\mathrm{N} 2$ neuraminidase and the Tamiflu-like ligands using alchemical perturbation methods. As a first step, we trialled multiple simulations protocols for their ability to produce stable neuraminidase complexes with ligands $\mathbf{1}$ and $\mathbf{2}$. Table 3 shows the conditions investigated, which varied the force field, water model, and the protein oligomerization state. From these simulations, we found that modelling the native tetramer was necessary for neuraminidase-ligand complex stability. In models containing only a single protomer, we saw conformational changes in the protein face that would have been stacked against an adjacent monomer, increased 150-loop flexibility and distortion of the active site residues. Specifically, the added flexibility to the 150-loop resulted in an increase in the 150-cavity volume and eventual displacement of the arginine residues in the binding site. The increased volume in the 150-cavity resulted in the displacement of $R_{2}$ (blue, Figure 2) from the 150-cavity within $50 \mathrm{~ns}$. This was true for every force field trialled, and was independent of the solvation model chosen. This observation is consistent with studies by Amaro et al. ${ }^{32}$ who proposed that the 150-loop is stabilised by two salt bridges between Asp147 and His 150, and between Asp147 and Arg-107 of the adjacent protein monomer. These salt bridges are observed by crystallography ${ }^{20}$ and were shown by the MD simulations of Amaro et al. to intermittently break and reform on a $100 \mathrm{~ns}$ time scale. The instability of the 150-loop in the monomer simulations can be rationalized by the loss of the stabilizing interaction between Asp-147 (Chain A) and Arg-107 (Chain D).

We also found that only the combination of the AMBER99SB-ILDN protein force field, the GAFF force field for the ligand and the four-point water TIP4P-ew water model produced stable protein/ligand models. Amaro et al. ${ }^{32}$ previously used the AMBER99SB force field to model the neuraminidase protein binding site. Masukawa et al. ${ }^{49}$ and Nguyen et al. ${ }^{31}$ have previously observed that retaining active site waters for an MD simulation is important for neuraminidase-ligand complex stability and for accurate binding free energy calculations. In this work, we found that the 3-point water models trialled did not produce stably bound ligands. However, the 4-point TIP4P-Ew model, which is optimized for the PME algorithm ${ }^{47}$ did produce stable protein/ligand complexes. It is possible that any 4-point water model would afford stably bound active site water molecules by long-timescale MD simulations, however we report the use of TIP4P-Ew here (Table 3). 
Table 3. Summary of simulation protocols evaluated to establish a method that gives stable neura minida se-ligand complexes.

\begin{tabular}{lllll}
\hline $\begin{array}{l}\text { Protein } \\
\text { oligomerization state }\end{array}$ & $\begin{array}{l}\text { Pigand force } \\
\text { field }\end{array}$ & $\begin{array}{l}\text { Water } \\
\text { model }\end{array}$ & $\begin{array}{l}\text { Protein-ligand } \\
\text { complex } \\
\text { stability }\end{array}$ \\
\hline Monomer & GROMOS54a7 & ATB & SPC/e & Low $^{\text {a }}$ \\
Monomer & OPLS-AA/L & LigParGen & TIP3P & Low \\
Monomer & CHARMM27 & CGenFF & TIP3P & Low \\
Monomer & AMBER99SB-ILDN 41 & GAFF & TIP3P & Low \\
Tetramer & GROMOS54a7 & ATB & SPC/e & Low \\
Tetramer & OPLS-AA/L & LigParGen & TIP3P & Low \\
Tetramer & CHARMM27 & CGenFF & TIP3P & Low \\
Tetramer & AMBER99SB-ILDN & GAFF & TIP3P & Moderate \\
Tetramer & OPLS-AA/L & LigParGen & TIP4P-Ew & Low \\
Tetramer & CHARMM27 & CGenFF & TIP4P-Ew & Low \\
Tetramer & AMBER99SB-ILDN & GAFF & TIP4P-Ew & High \\
\hline
\end{tabular}

${ }^{\mathrm{a}}$ Key interactions between the protein and ligand break, and ligand dissociates within $50 \mathrm{~ns}$. ${ }^{\mathrm{b}}$ Ligand dissociation occurs between 50 to $350 \mathrm{~ns} .{ }^{\mathrm{c}}$ Complex remains stable for $350 \mathrm{~ns}$.

Figure 4 shows the RMSD of the protein backbone, binding site arginine residues and ligand during the $350 \mathrm{~ns}$ simulations of Tamiflu carboxylate (1) and GlcA analogue (2) bound to N2 neuraminidase. The movement of $\mathbf{1}$ is measured relative to the crystal structureand the movement of GlcA $\mathbf{2}$ is measured relative to the synthetic starting model. In both simulations, the protein heavy atom RMSD converged to $1.4 \AA$ RMSD from the original crystal structure (Figure 4A) and the binding site arginine residues were stable, converging to $1.0 \AA$ A RMSD (Figure 4B). In both cases, both the active site arginine cluster (Arg-118, Arg-272 and Arg-371) as well as Arg-224 did not move significantly from the crystal structure. The ligand atoms converged to $0.6 \AA$ and $2.0 \AA$ RMSD from crystal structures, for complexes with $\mathbf{1}$ and $\mathbf{2}$, respectively (Figure $4 \mathrm{C}$ ).

These results presented in Table 3 and Figure 4 improve on the findings of Ren et al., ${ }^{34}$ who investigated 16 different protein and water forcefield combinations to determine the best MD parameters for $20 \mathrm{~ns}$ simulations subject to MM-PBSA analysis. These authors also reported that the AMBER forcefield variants result in the most accurate binding free energy calculations by MM-PBSA, consistent with data in Table 3 and Figure 4. Ren et al. detail the use of monomeric neuraminidase simulation systems with 3-point water models. From the data presented in Table 3, we suggest that Ren et al. would observe dissociation of the protein-ligand complexes in their simulation systems on longer timescales than $20 \mathrm{~ns}$. We show here an optimal MD protocol for simulating N2 neuraminidase-ligand complexes. 

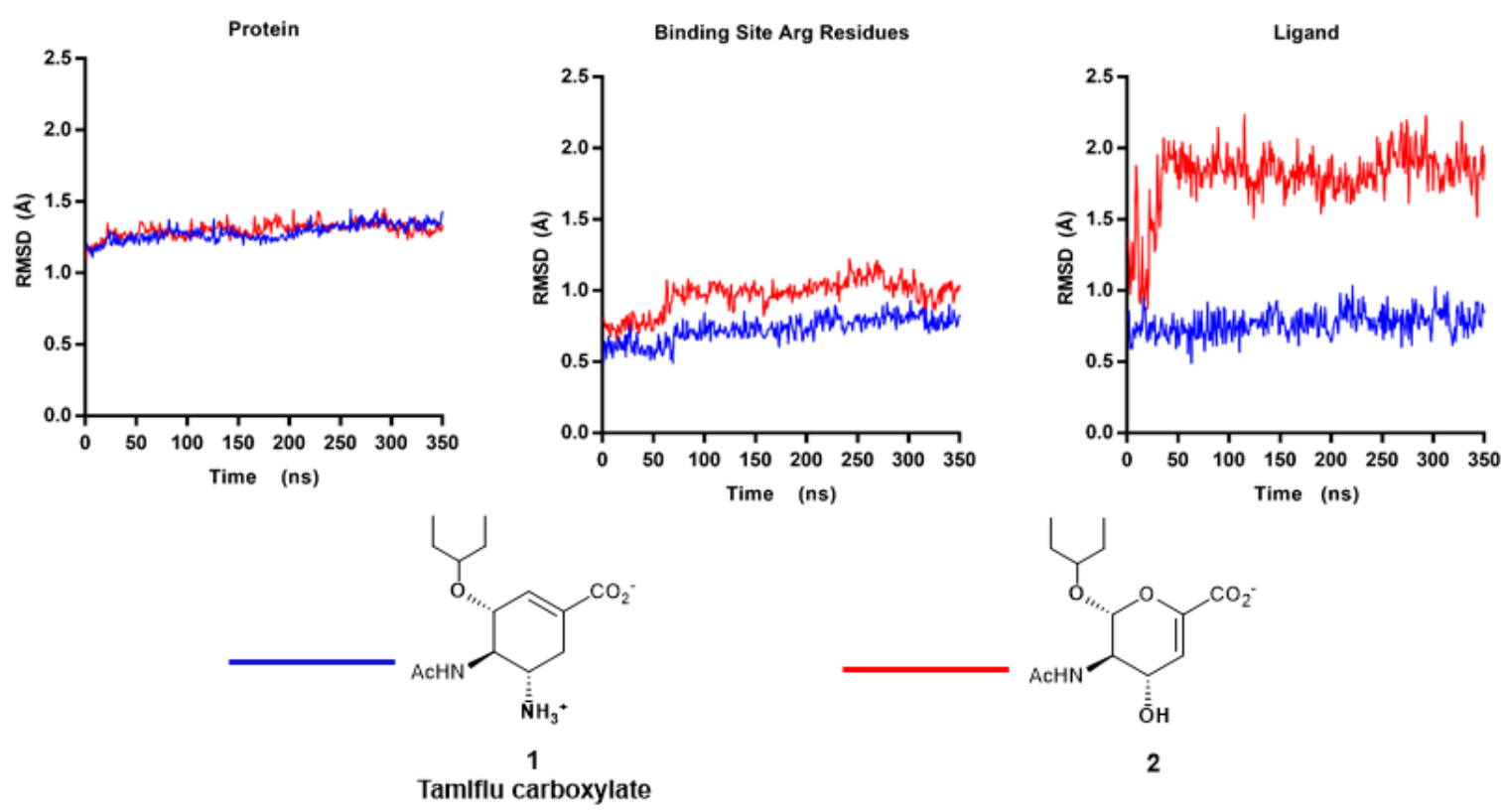

Figure 4. RMSD relative to crystal pose over simulation time for neuraminidase in complex with Tamiflu carboxylate (blue) and 2 (red). (A) Plots of protein heavy atom RMSD for tetrameric neuraminidase.(B) RMSD of Arg residues within $5 \AA$ of the bound ligand. (C) Ligand RMSD (right).

To determine how well relative alchemical perturbation could predict differences in binding free energy $(\Delta \Delta G)$ for neuraminidase ligands, we performed a set of 18 alchemical perturbations for ligands 1-9 (detailed in supplementary information Table S1). Four transformations produced unrealistically large $\Delta \Delta G$ values, which were discarded, leaving set of 14 perturbations connecting 9 compounds. The resulting perturbation map is shown in Figure 5. In performing the calculations, we found that transformations involving the charged ammonium group of compound 1a were generally problematic. It was, however, possible to transform $\mathbf{1 a}$ into other compounds through intermediate $\mathbf{1} \mathbf{b}$, which contains a neutral amino group. The transformations formed a number of closed cycles. An in-house Python script identified a total of 15 closed cycles in the perturbation map. Hysteresis correction was performed by cycle closure across the perturbation map using the method described by Wang et $a l^{20}$ and raw values of $\Delta \Delta G$ were corrected to make all closed cycles sum to $0 \mathrm{~kJ} \mathrm{~mol}^{-1}$ in the statistically most likely way, given the assumption that the error in every perturbation is the same. The cycle with thelargest hysteresis was: $1 \mathrm{~b} \rightarrow \mathbf{2} \rightarrow \mathbf{4} \rightarrow \mathbf{6} \rightarrow \mathbf{9} \rightarrow \mathbf{1 b}$ where, instead of affording a $\Delta \Delta G$ value of $0 \mathrm{~kJ} \mathrm{~mol}^{-1}$, the closed cycle had a hysteresis of $84.4 \mathrm{~kJ} \mathrm{~mol}^{-1}$. This cycle is large and contains transformations between ligands with the chemically different Tamiflu and GlcA-based scaffolds. The hysteresis correction algorithm reduced the hysteresis in each of the 15 closed cycles to $0 \mathrm{~kJ} \mathrm{~mol}^{-1}$. 


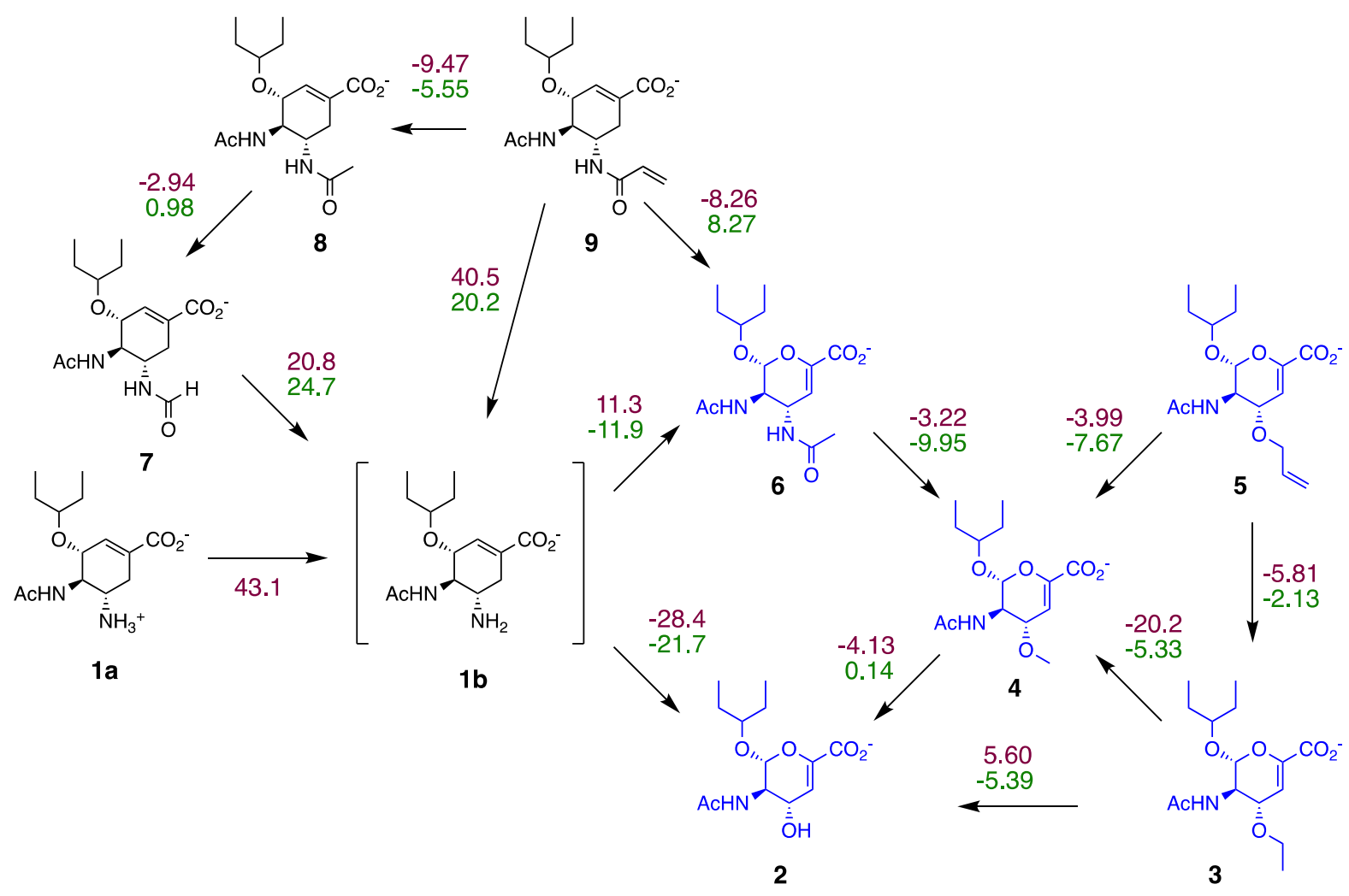

Figure 5. Perturbation map for relative alchemical transformations between ligands based on Tamiflu carboxylate (1) and the GlcA cores. Raw $\Delta \Delta G$ values are shown in red text (top value). $\Delta \Delta G$ values corrected by the hysteresis correction algorithm are shown in green (bottom value). Structures with a Tamiflu scaffold are in black and GlcA analogues are in blue. Structures $\mathbf{1 a}$ and $\mathbf{1 b}$ differ in the protonation state of the amino group.
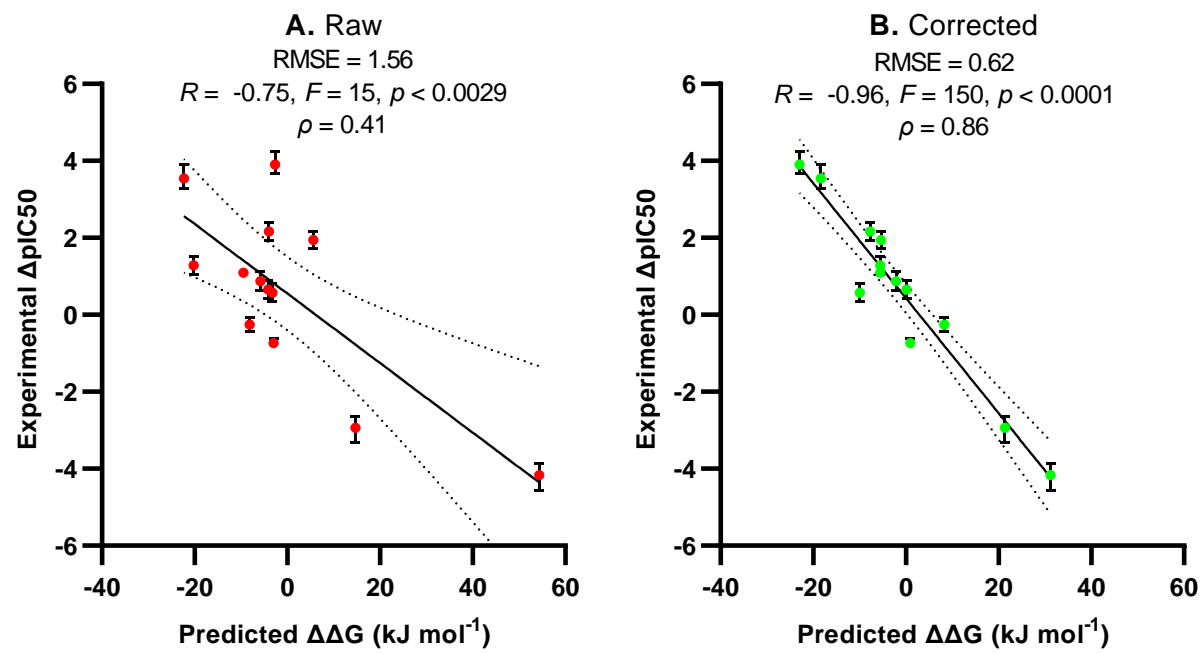

Figure 6. (A) Raw predicted $\Delta \Delta \mathrm{G}$ aga inst experimental $\Delta \mathrm{pIC} 50$. (B) Predicted $\Delta \Delta \mathrm{G}$ va lues a ga inst experimental $\Delta \mathrm{pIC} 50$ after correcting for hysteresis in the predicted $\Delta \Delta \mathrm{G}$ values using the method by Wang et al. ${ }^{20}$ Errors bars representing $\pm 2 \mathrm{SD}$ in the experimental IC50 are shown. Lines of best fit from linear regression are shown as a solid line. The $95 \%$ CI limits of the two lines of best fit are shown as dashed lines. The RMSE values represent the a verage residual in predicted $\triangle \mathrm{pIC} 50$ from the line of best fit. 
The predicted $\Delta \Delta G$ values before and after hysteresis correction, were correlated with experimental $\Delta \mathrm{pIC50}$ values (Figure 6). The raw predicted $\Delta \Delta G$ values (before hysteresis correction) have modest predictive value and correlate with $\Delta$ pIC50 with Pearson's $R=-0.75$ and Spearman's $\rho=0.41$ (Figure $6 \mathrm{~A}$ ). By correcting for hysteresis (Figure 6B) the correlation between predicted $\Delta \Delta G$ and $\Delta$ pIC50 values improves to $R=-0.96$ and $\rho=0.86$. Thus, hysteresis correction substantially improved correlation to experiment. The corrected $\rho^{2}$ value of 0.74 means that approximately 74 percent of the variance in the rank order of differences in experimental pIC50 can be explained by the modelling. Further, the $95 \% \mathrm{CI}$ in the line of best fit between raw $\Delta \Delta G$ predictions and experimental $\Delta$ pIC50 values was considerably broader than when the hysteresis correction was performed (Figure 6A and6B). A total of six datapoints are outside this $95 \% \mathrm{CI}$ of the line of best fit in Figure 6A, while two are outside the narrower 95\% CI in the line of best fit in Figure 6B. This decrease in both the number of predictions outside of the 95\% $\mathrm{CI}$ in the line of best fit, and in the range in each line of best fit, indicates the improved predictive power of our model after the hysteresis correction is applied to the raw data. The RMSE between experimental and predicted $\triangle \mathrm{pIC} 50$ values from our linear regression model was 1.56 and 0.62 when using the raw and corrected data for the line of best fit (Figures $6 \mathrm{~A}$ and 6B). These RMSE values represent the average residual in $\triangle \mathrm{pIC} 50$ from the line of best fit. By comparison with experimental errors, the range in the experimental pIC50 value for Tamiflu carboxylate (1) was 0.46 , representing \pm 2 SD in the IC50 value. Ourbinding free energy calculations are therefore reliable in predicting which compounds more strongly inhibit neuraminidase in hit-to-lead and lead-optimisation projects, approaching the accuracy of experiment. This level of accuracy is similar to that previously reported for binding free energy calculations with neuraminidase ligands. For example, Mai et al..$^{28}$ used steered MD to estimate ligand binding strength for a similar sample size of neuraminidase ligands and obtained a correlation coefficient of $R=0.97$ when comparing $F_{\max }$ with experimental $\Delta G$.

\section{CONCLUSION}

We have established a set of computational conditions that are suitable for modelling ligand binding to influenza virus neuraminidase. It has been shown that neuraminidase must be simulated as the native tetramer for stable complexes. It is also shown that AMBER99SB-ILDN and GAFF force fields, with the TIP4P-Ew water model, afforded the most stable N2 neuraminidase complexes by MD simulation over a $350 \mathrm{~ns}$ timescale used here. We used alchemical perturbation with BAR to predict the relative binding affinities $(\Delta \Delta G)$ of a set of 9 neuraminidase inhibitors. The raw perturbation data was corrected for hysteresis, which greatly improved the correlation with experimental data. After hysteresis correction, we found a correlation between $\Delta \Delta G$ and $\Delta$ pIC50 values, with $R=-0.96$ for a total of 14 transformations. The RMSE in $\triangle \mathrm{pIC50}$ values from the linear regression model on the corrected $\Delta \Delta G$ values was 0.62 , approaching the range in the pIC50 values from repeated experimental measurements, which was 0.46 for Tamiflu carboxylate (given by \pm 2 SD in the IC50 value). Therefore, the level of error in our models approaches the experimental error of the original assays. Additionally, there are 
differences in how previous modelling studies have treated neuraminidase, with force field choices varying as either CHARMM27, OPLS, GROMOS96 or AMBER99SB, and either 3-point or 4-point water models used to model solvent. We report here an optimal MD protocol. These MD methods also have potential applications for homologous glycohydrolases. We anticipate that these methods for molecular dynamics simulations and binding free energy calculations can be applied in the future to assist with the discovery of new drugs targeting neuraminidase-like proteins. Examples where our MD protocol and perturbation methods could potentially be applied are for ligands binding to: the homologous haemagglutinin-neuraminidase $(\mathrm{HN})$ from human parainfluenza virus,${ }^{50-52}$ bacterial neuraminidase $s^{53}$ or the putative pseudaminidase reported in 2008 by Xu et al.,${ }^{54}$ which is thought to cleave pseudaminic acid ${ }^{55}$ from Gram negative bacteria cell-surfaces and to produce biofilm.

\section{ACKNOWLEDGMENTS}

We acknowledge the MASSIVE and NCI supercomputing facilities for computational resources and thank them for technical support. BJW-N gratefully thanks the Australian government for his research training program (RTP) grant. Discussions with Jeff Dyason and Assoc. Prof. Milton Kiefel of the Griffith University Institute for Glycomics have been very helpful in this work, and we offer them our thanks.

\section{REFERENCES}

1. Patel, H.; Kukol, A., Integrating molecular modelling methods to advance influenza A virus drug discovery. Drug Discov. 2020.

2. Copeland, R. A.; Pompliano, D. L.; Meek, T. D., Drug-target residence time and its implications for lead optimization. Nat. Rev. Drug Discov. 2006, 5 (9), 730.

3. Zwanzig, R. W., High - temperature equation of state by a perturbation method. I. nonpolar gases. J. Chem. Phys. 1954, 22 (8), 1420-1426.

4. Williams-Noonan, B. J.; Yuriev, E.; Chalmers, D. K., Free Energy Prediction for Binding of Allosteric Inhibitors of Human Immunodeficiency Virus-1 Integrase: a Comparative Study. Submitted 2020.

5. Williams-Noonan, B. J.; Yuriev, E.; Chalmers, D. K., Free Energy Methods in Drug Design: The Prospects of 'Alchemical Perturbation'in Medicinal Chemistry. J. Med. Chem. 2017.

6. Perez-Benito,L.; Casajuana-Martin, N.; Jimenez-Roses, M.; van Vlijmen, H.; Tresadern, G., Predicting Activity Cliffs with Free-Energy Perturbation. J. Chem. Theory Comput. 2019, 15 (3 ), 18841895.

7. Roos, K.; Wu, C.; Damm, W.; Reboul, M.; Stevenson, J. M.; Lu, C.; Dahlgren, M. K.; Mondal, S.; Chen, W.; Wang, L., OPLS3e: Extending force field coverage fordrug-like small molecules. J. Chem. Theory Comput. 2019, 15 (3), 1863-1874.

8. Taubenberger, J. K.; Morens, D. M., 1918 Influenza: the mother of all pandemics. Revista Biomed. 2006, 17 (1), 69-79.

9. Alexander, D., Avian influenza viruses and human health. Dev. Biol. 2006, 124, 77-84.

10. von Itzstein, M.; Wu, W.-Y.; Kok, G. B.; Pegg, M. S.; Dyason, J. C.; Jin, B.; Van Phan, T.; Smythe, M. L.; White, H. F.; Oliver, S. W., Rational design of potent sialidase-based inhibitors of influenza virus replication. Nature 1993, 363 (6428), 418. 
11. Varghese, J. N.; Epa, V. C.; Colman,P. M., Three-dimensional structure of the complex of 4guanidino-Neu5Ac2en and influenza virus neuraminidase. Protein science : a publication of the Protein Society 1995, 4 (6), 1081-7.

12. Rohloff, J. C.; Kent, K. M.; Postich, M. J.; Becker, M. W.; Chapman, H. H.; Kelly, D. E.; Lew, W.; Louie, M. S.; McGee, L. R.; Prisbe, E. J., Practical total synthesis of the anti-influenza drug GS-4104. J. Org. Chem. 1998, 63 (13), 4545-4550.

13. Sidwell, R. W.; Huffman, J. H.; Barnard, D. L.; Bailey, K. W.; Wong, M.-H.; Morrison, A.; Syndergaard, T.; Kim, C. U., Inhibition of influenza virus infections in mice by GS4104, an orally effective influenza virus neuraminidase inhibitor. Antivir. Res. 1998, 37 (2), 107-120.

14. Li, W.; Escarpe, P. A.; Eisenberg, E. J.; Cundy, K. C.; Sweet, C.; Jakeman, K. J.; Merson, J.; Lew, W.; Williams, M.; Zhang, L., Identification of GS 4104 as an orally bioavailable prodrug of the influenza virus neuraminidase inhibitor GS 4071. Antimicrob. Agents Chemother. 1998, 42 (3), 647653.

15. Lew, W.; Chen, X.; Kim, C. U., Discovery and development of GS 4104 (oseltamivir) an orally active influenza neuraminidase inhibitor. Curr. Med. Chem. 2000, 7 (6), 663-672.

16. Webster, R. G.; Bean, W. J.; Gorman, O. T.; Chambers, T. M.; Kawaoka, Y., Evolution and ecology of influenza A viruses. Microbiol. Mol. Biol. Rev. 1992, 56(1), 152-179.

17. Yoon, S.-W.; Webby, R. J.; Webster, R. G., Evolution and ecology of influenza A viruses. In Influenza Pathogenesis and Control-Volume I, Springer: 2014; pp 359-375.

18. Colman, P. M.; Varghese, J.; Laver, W., Structure of the catalytic and antigenic sites in influenza virus neuraminidase. Nature 1983, 303 (5912), 41.

19. Bhatt, B.; Böhm, R.; Kerry, P. S.; Dyason, J. C.; Russell, R. J.; Thomson, R. J.; von Itzstein, M., Exploring the interactions of unsaturated glucuronides with influenza virus sialidase. J. Med. Chem. 2012, $55(20), 8963-8968$.

20. Wang, L.; Deng, Y.; Knight, J. L.; Wu, Y.; Kim, B.; Sherman, W.; Shelley, J. C.; Lin, T.; Abel, R., Modeling local structural rearrangements using FEP/REST: application to relative binding affinity predictions of CDK2 inhibitors. J. Chem. Theory Comput. 2013, 9 (2), 1282-93.

21. Varghese, J. N.; McKimm - Breschkin, J. L.; Caldwell, J. B.; Kortt, A. A.; Colman, P. M., The structure of the complex between influenza virus neuraminidase and sialic acid, the viral receptor. Proteins 1992, 14 (3), 327-332.

22. Gubareva, L. V.; Webster, R. G.; Hay den, F. G., Comparison of the activities of zanamivir, oseltamivir, and RWJ-270201 against clinical isolates of influenza virus and neuraminidase inhibitorresistant variants. Antimicrob. Agents Chemother. 2001, 45 (12), 3403-3408.

23. Wang, K.; Yang, F.; Wang, L.; Liu, K.; Sun, L.; Lin, B.; Hu, Y.; Wang, B.; Cheng, M.; Tian, Y., Synthesis and biological evaluation of $\mathrm{NH} 2$-acyl oseltamivir analogues as potent neuraminidase inhibitors. Eur. J. Med. Chem. 2017, 141, 648-656.

24. Taylor, N. R.; von Itzstein, M., Molecular Modeling Studies on Ligand Binding to Sialidase from Influenza Virus and the Mechanism of Catalysis. J. Med. Chem. 1994, 37 (5), 616-624.

25. Lawrenz, M.; Baron, R.; McCammon, J. A., Independent-trajectories thermodynamicintegration free-energy changes for biomolecular systems: determinants of $\mathrm{H} 5 \mathrm{~N} 1$ avian influenza virus neuraminidase inhibition by peramivir. J. Chem. Theory Comput. 2009, 5 (4), 1106-1116.

26. Lawrenz, M.; Wereszczynski, J.; Amaro, R.; Walker, R.; Roitberg, A.; McCammon, J. A., Impact of calcium on $\mathrm{N} 1$ influenza neuraminidase dynamics and binding free energy. Proteins 2010, 78 (11), 2523-2532.

27. Kiso, M.; Kubo, S.; Ozawa, M.; Le, Q. M.; Nidom, C. A.; Yamashita, M.; Kawaoka, Y., Efficacy of the new neuraminidase inhibitor CS-8958 against H5N1 influenza viruses. PLOS Pathog. 2010, 6 (2), e1000786. 
28. Mai, B. K.; Li, M. S., Neuraminidase inhibitor R-125489-a promising drug for treating influenza virus: steered molecular dynamics approach. Biochem. Biophys. Res. Commun. 2011, 410 (3), 688-691.

29. Yamashita, M.; Tomozawa, T.; Kakuta, M.; Tokumitsu, A.; Nasu, H.; Kubo, S., CS-8958, a prodrug of the new neuraminidase inhibitor R-125489, shows long-acting anti-influenza virus activity. Antimicrob. Agents Chemother. 2009, 53 (1), 186-192.

30. Watanabe, A.; Chang, S.-C.; Kim, M. J.; Chu, D. W.-s.; Ohashi, Y.; Group, M. S., Longacting neuraminidase inhibitor laninamivir octanoate versus oseltamivir for treatment of influenza: a double-blind, randomized, noninferiority clinical trial. Clin. Infect. Dis. 2010, 51 (10), 1167-1175.

31. Nguyen, T. T.; Viet, M. H.; Li, M. S., Effects of water models on binding affinity: evidence from all-atom simulation of binding of tamiflu to A/H5N1 neuraminidase. Sci. World J. 2014, 2014.

32. Amaro, R. E.; Swift, R. V.; Votapka, L.; Li, W. W.; Walker, R. C.; Bush, R. M., Mechanism of 150-cavity formation in influenza neuraminidase. Nat. Commun. 2011, 2, 388.

33. Jusoh, N.; Zainal, H.; Hamid, A. A. A.; Bunnori, N. M.; Abd Halim, K. B.; Abd Hamid, S., In silico study of carvone derivatives as potential neuraminidase inhibitors. J. Mol. Model. 2018, 24 (4), $1-13$.

34. Ren, J.; Yuan, X.; Li, J.; Lin, S.; Yang, B.; Chen, C.; Zhao, J.; Zheng, W.; Liao, H.; Yang, Z., Assessing the performance of the g_mmpbsa tools to simulate the inhibition of oseltamivir to influenza virus neuraminidase by molecular mechanics Poisson-Boltzmann surface area methods. $J$. Chin. Chem. Soc. 2020, 67 (1), 46-53.

35. Hanpaibool, C.; Leelawiwat, M.; Takahashi, K.; Rungrotmongkol, T., Source of oseltamivir resistance due to single E119D and double E119D/H274Y mutations in pdm09H1N1 influenza neuraminidase. J. Comput. Aided Mol. Des. 2020, 34 (1), 27-37.

36. Mahalapbutr, P.; Sangkhawasi, M.; Kammarabutr, J.; Chamni, S.; Rungrotmongkol, T., Rosmarinic acid as a potent influenza neuraminidase inhibitor: In vitro and in silico study. Curr. Top. Med. Chem. 2020.

37. Kato, Y.; Takahashi, K.; Ito, F.; Suzuki, S.; Fukui, K.; Mimaki, M.; Suzuki, K., Novel oseltamivir-resistant mutations distant from the active site of influenza B neuraminidase. J. Biomol. Struct. Dyn. 2020, 1-10.

38. Bell, J.; Cao, Y.; Gunn, J.; Day, T.; Gallicchio, E.; Zhou, Z.; Levy, R.; Farid, R., PrimeX and the Schrödinger computational chemistry suite of programs. 2006; pp 534-538.

39. Tribello, G. A.; Bonomi, M.; Branduardi, D.; Camilloni, C.; Bussi, G., PLUMED 2: New feathers for an old bird. Comput. Phys. Commun. 2014, 185 (2), 604-613.

40. Abraham, M.; van der Spoel, D.; Lindahl, E.; Hess, B., the GROMACS development team, GROMACS User Manual version 5.1. 2, 2016.

41. Lindorff - Larsen, K.; Piana, S.; Palmo, K.; Maragakis, P.; Klepeis, J. L.; Dror, R. O.; Shaw, D. E., Improved side - chain torsion potentials for the Amber ff99SB protein force field. Proteins 2010, 78 (8), 1950-1958.

42. Sprenger, K.; Jaeger, V. W.; Pfaendtner, J., The general AMBER force field (GAFF) can accurately predict thermodynamic and transport properties of many ionic liquids. J. Phys. Chem. B 2015, 119 (18), 5882-5895.

43. de Leeuw, S. W.; Perram, J. W.; Smith, E. R. In Simulation of electrostatic systems in periodic boundary conditions. I. Lattice sums and dielectric constants, Proc. Royal Soc. Lond. A, The Royal Society: 1980; pp 27-56.

44. Verlet, L., Computer "experiments" on classical fluids. I. Thermodynamical properties of Lennard-Jones molecules. Phys. Rev. 1967, 159 (1), 98. 
45. Hess, B.; Bekker, H.; Berendsen, H. J.; Fraaije, J. G., LINCS: a linear constraint solver for molecular simulations. J. Comput. Chem. 1997, 18 (12), 1463-1472.

46. Darden, T.; York, D.; Pedersen, L., Particle mesh Ewald: An N· $\log (\mathrm{N})$ method for Ewald sums in large systems. J. Chem. Phys. 1993, 98 (12), 10089-10092.

47. Horn, H. W.; Swope, W. C.; Pitera, J. W.; Madura, J. D.; Dick, T. J.; Hura, G. L.; HeadGordon, T., Development of an improved four-site water model for biomolecular simulations: TIP4PEw. J. Chem. Phys. 2004, 120 (20), 9665-9678.

48. Loeffler, H. H.; Michel, J.; Woods, C., FESetup: automating setup for alchemical free energy simulations. J. Chem. Inf. Model. 2015.

49. Masukawa, K. M.; Kollman, P. A.; Kuntz, I. D., Investigation of Neuraminidase-Substrate Recognition Using Molecular Dynamics and Free Energy Calculations. J. Med. Chem. 2003, 46 (26), 5628-5637.

50. Spriggs, M.; Murphy, B.; Prince, G.; Olmsted, R.; Collins, P., Expression of the F and HN glycoproteins of human parainfluenza virus type 3 by recombinant vaccinia viruses: contributions of the individual proteins to host immunity. J. Virol. 1987, 61 (11), 3416-3423.

51. Winger, M.; von Itzstein, M., Exposing the flexibility of human parainfluenza virus hemagglutinin-neuraminidase. J. Am. Chem. Soc. 2012, 134 (44), 18447-18452.

52. Guillon, P.; Dirr, L.; El-Deeb, I. M.; Winger, M.; Bailly, B.; Haselhorst, T.; Dyason, J. C.; Von Itzstein, M., Structure-guided discovery of potent and dual-acting human parainfluenza virus haemagglutinin-neuraminidase inhibitors. Nature Commun. 2014, 5, 5268.

53. Gaskell, A.; Crennell, S.; Taylor, G., The three domains of a bacterial sialidase: a $\beta$-propeller, an immunoglobulin module and a galactose-binding jelly-roll. Structure 1995, 3 (11), 1197-1205.

54. Xu, G.; Ryan, C.; Kiefel, M. J.; Wilson, J. C.; Taylor, G. L., Structural studies on the Pseudomonas aeruginosa sialidase-like enzyme PA2794 suggest substrate and mechanistic variations. J. Mol. Biol. 2009, 386 (3), 828-840.

55. Zunk, M.; Williams, J.; Carter, J.; Kiefel, M. J., A new approach towards the synthesis of pseudaminic acid analogues. Org. Biomol. Chem. 2014, 12 (18), 2918-2925. 